\title{
Efficiency of intrauterine insemination (IUI) along with different ovarian stimulation protocols as a method of treating infertility
}

\author{
Jalpa K. Bhatt \\ Associate Professor, Dept. of Obstetrics and Gynaecology, Dr M.K. Shah Medical College and Research Center, Chandkheda, \\ Ahmedabad, India
}

*Corresponding Author:

Email: drjalpavyas@yahoo.com

\begin{abstract}
Introduction: Infertility affects $8-12 \%$ couples during their reproductive life. The number of infertile couple is on rise in India. The common reason for infertility may be female factor, male factor, unexplained or combined. IUI is a frequently indicated therapeutic modality for couples with male factor infertility, unexplained infertility and women with cervical mucus problems and mild endometriosis.

Materials and Methods: This is a retrospective study performed at fertility clinic. The patients attending the outdoor department for infertility treatment, those who underwent ovarian stimulation along with IUI cycles were enrolled in the study.

Results: Maximum 55 (52.88\%) patients were of 26-30 years age group. 85(81.73\%) Participants were having complain of primary infertility whereas $19(18.26 \%)$ were having secondary infertility.

Out of 30 conceptions achieved, 18 (60\%) were stimulated by gonadotrophins along with IUI.

Conclusion: IUI along with controlled ovarian stimulation gives higher pregnancy rate.
\end{abstract}

Keywords: Ovarian stimulation, Infertility, Artificial reproductive techniques, Intrauterine insemination.

\section{Introduction}

Human reproduction is considered weaker in comparison to other animals. Infertility is a worldwide problem. The World Health Organization defines infertility as follows ${ }^{1}$ :

"Infertility is a disease of the reproductive system defined by the failure to achieve a clinical pregnancy after 12 months or more of regular unprotected sexual intercourse (and there is no other reason, such as breastfeeding or postpartum amenorrhoea). Primary infertility is infertility in a couple who have never had a child. Secondary infertility is failure to conceive following a previous pregnancy. Infertility may be caused by infection in the man or woman, but often there is no obvious underlying cause."

According to WHO," infertility affecting 812 percent couple $(50-80$ million $)$ during their reproductive lives (WHO, 1991). Childlessness has serious demographic, social and health implications. The ease with which women can be labelled infertile or resist the label, the experiences of childless women and the process of seeking solutions for infertility all go beyond the biological fact of reproductive impairment "(Unisa, 1999; Pearce, 1999). ${ }^{2}$

India accounts for 5-10million of the infertile couples, and the number is increasing at the rate of $5 \%$ every 2 years. ${ }^{3}$ The WHO estimates the overall prevalence of primary infertility in India to be between $3.9 \%$ and $16.8 \% .^{4}$

Infertility is a social problem in India. Couples face tremendous personal sufferings and mental stress when conception becomes difficult due to social pressure and stigma of being infertile. The couple becomes very anxious to conceive and in the agony many a time's females develop subclinical depression as they are at more social pressure and more emotional instability. The couple faces many barriers even for seeking treatment and affordability may prove a major issue. Many couples face problems in married life too as they take it as a major crisis in their life.

"Etiology of infertility varies from one region to another and from one population to another. The incidence of infertility are estimated from $12 \%$ to 28 $\% .{ }^{5}$ Out of it $20-30 \%$ of infertility cases are due to male infertility, 20-35\% are due to female infertility, and 25$40 \%$ are due to combined problems in both parts". ${ }^{6}$ In $10-20 \%$ of cases, unexplained and unknown causes. Due to lifestyle changes and environmental effects incidence of infertility is on rise.

"Intrauterine insemination (IUI) is a frequently indicated therapeutic modality in infertility. This procedure is used for couples with unexplained infertility, minimal male factor infertility, and women with cervical mucus problems. In the past few years, there was increased interest in the use of IUI after ovarian stimulation and pregnancy rate was significantly better than the natural cycle"., 7

IUI is a technique by which we place washed sperm inside a woman's uterus. "The purpose of IUI is to increase the density of sperm nearer to ovum lying in fallopian tubes and resultant increase the chance of fertilization. IUI can be done on a natural cycle but preferably should be combined with a stimulated cycle to get good results. Artificial insemination has mainly been used to treat unexplained infertility (usually combined with super ovulation) and male factor infertility." It is also a preferred therapeutic modality for mild endometriosis and ovarian factor infertility. 
IUI is the best studied and most widely practiced of all the insemination techniques". ${ }^{10}$

The chances of success in a IUI cycle depends on many different factors. If a couple undergoes regular IUI cycles successive months, the chances of conception may be as high as $20 \%$ per cycle. Other factors like age of patient, the factor for infertility, protocol for ovarian stimulation also plays a role in success.

Before IUI, the woman should be investigated for any hormonal imbalance, infection or any structural problems. However, the patients with damaged fallopian tubes, pelvic inflammatory diseases and moderate and severe endometriosis should not be offered IUI.

\section{Materials and Methods}

The study was carried out at Shivanjali women's hospital, Ahmedabad, Gujarat, India. This is a retrospective study. The patients attending the outpatient department of fertility clinic at shivanjali women's hospital were examined and investigated for infertility. Out of them 104 couples were suggested IUI for different indications. The couples were counselled about the treatment and with the couple's consent, treatment initiated. Total 104 couples underwent 278 IUI cycles with different regimes for ovarian stimulation. All 104 couples were enrolled in the study. All IUI cycles were carried out between 2008 to 2016 at shivanjali women's clinic.

In an IUI cycle, the couple is advised to report on day 2 of period. Before starting ovarian stimulation, a base line transvaginal ultrasound scan (TVS) was performed to check pelvic organs.

The stimulation was done by either Tab. Clomiphene Citrate 50/100 mg per day or Tab.Letrozole $2.5 \mathrm{mg}$ for 5 days from day 2 to 6 and/ or gonadotrophins on day 6 and 8 and then as per requirement of the particular patient. Monitoring of ovarian as well as endometrial responses to the drugs was done alternate days by transvaginal ultrasound from day 8 .

Human chorionic gonadotropin (HCG) 5000 IU IM/SC was given to induce ovulation when leading follicle diameter reaches the size of $18 \mathrm{~mm}-23 \mathrm{~mm}$ depending on the method of stimulation. After 34 to 36 hours of HCG administration single IUI was done.

For Preparation of semen sample, husband was informed to submit a semen sample in a sterile container approximately two hours before IUI. The semen was allowed to liquefy. A semen analysis done and method selected for preparation. Swim up technique or density gradient method was used in our lab according to sperm count and motility in provided sample. Once the sample was ready, patient was taken in lithotomy position and IUI done under proper aseptic precaution. Progesterone was given to support the luteal phase. When patient had delayed period pregnancy was checked with urine pregnancy test. All conceptions were confirmed with ultra-sonography of intrauterine pregnancy.

\section{Results}

Table 1: Age group of the patients

\begin{tabular}{|l|c|}
\hline $\begin{array}{c}\text { Age of the patients } \\
\text { undergoing treatment }\end{array}$ & $\begin{array}{c}\text { No. of patients } \\
(\mathbf{N}=104)\end{array}$ \\
\hline $21-25$ & $17(16.34 \%)$ \\
\hline $26-30$ & $55(52.88 \%)$ \\
\hline $31-35$ & $24(23.07 \%)$ \\
\hline $36-40$ & $8(7.69 \%)$ \\
\hline
\end{tabular}

Table 2: Type of infertility ( $N=104)$

\begin{tabular}{|c|c|}
\hline Primary & Secondary \\
\hline $85(81.73 \%)$ & $19(18.26 \%)$ \\
\hline
\end{tabular}

Table 3: Duration of infertility ( $N=104)$

\begin{tabular}{|l|c|}
\hline \multicolumn{1}{|c|}{$<$ years } & $\mathbf{2 0}(\mathbf{1 9 . 2 3 \%})$ \\
\hline $2-5$ years & $46(44.23 \%)$ \\
\hline $5-10$ years & $28(26.92 \%)$ \\
\hline$>\mathbf{1 0}$ years & $\mathbf{1 0}(\mathbf{9 . 6 1 \%})$ \\
\hline
\end{tabular}

Table 4: Factors of infertility

\begin{tabular}{|l|c|c|c|}
\hline Male & Female & Combined & Unexplained \\
\hline 25 & 59 & 12 & 8 \\
\hline
\end{tabular}

Table 5: Fate of conception $(\mathrm{N}=30)$

\begin{tabular}{|l|c|}
\hline Full term pregnancy & 22 \\
\hline abortion & 7 \\
\hline Ectopic pregnancy & 1 \\
\hline
\end{tabular}

Table 6: Conception with different modalities of ovarian stimulation

\begin{tabular}{|l|c|}
\hline $\begin{array}{c}\text { Conceived with } \\
\text { modality of ovarian } \\
\text { stimulation } \\
\text { combined with IUI }\end{array}$ & $\begin{array}{c}\text { No of patients } \\
(\mathbf{N}=30)\end{array}$ \\
\hline Clomiphene citrate & $7(23.33 \%)$ \\
\hline Letrozole & $5(16.66 \%)$ \\
\hline $\begin{array}{l}\text { Clomiphene/Letrozole } \\
\text { +gonadotrophins }\end{array}$ & $18(60 \%)$ \\
\hline
\end{tabular}

\section{Discussion}

21-25 years there were $17(16.34 \%)$ patients, 2630years 55(52.88\%) patients, 31-35 years $24(23.07 \%)$ patients and in 36-40 years age group $8(7.69 \%)$ patients. Fecundity decreases markedly after the age of 35 years in women .Late marriages and delaying pregnancy contributes to major part of the problem of infertility. As women are more and more opting for higher studies and having good carrier options these days, this is quite common. In our study more than $30 \%$ (32) of patients are above age of 30 years.

Out of 104 participants, 85 participants complained of primary infertility and 19 secondary infertility. 
Twenty (19.23\%) patients were having less than 2 years of infertility. Majority of patients that is $46(44.23 \%)$ were having 2-5 years of infertility. In 5-10 years of infertility we have $28(26.92 \%)$ patients. Ten (9.61\%) patients were having more than 10 years of infertility. Socioeconomic condition, religious beliefs and lack of knowledge may be responsible for delay in seeking proper medical help for the problem.

Out of 104 couples, 59 had female factor infertility, 25 had male factor infertility, 12 had combined and 8 couples had unexplained infertility. Categories of etiologies of female infertility in study group were ovarian including polycystic ovaries $(\mathrm{N}=65)$, cervical factor $(\mathrm{N}=2)$, mild endometriosis $(\mathrm{N}=2)$, vaginismus $(\mathrm{N}=2)$. Environmental factors, change in lifestyle and dietary habits is responsible for increased incidence of male and female infertility. Habits like smoking, tobacco, alcohol also contributes to high incidence of male factor infertility.

In our study, out of 104 patients and 278 IUI cycles we had achieved 30 conceptions. Out of them 7 aborted, 1 ectopic pregnancy and the rest 22 conceptions reached up to full term pregnancies. All were singleton pregnancies. Seven $(23.33 \%)$ patients were induced with Clomephene Citrate for IUI cycle, $5(16.66 \%)$ with Letrozole and $18(60 \%)$ patients were induced by oral Clomephene/Letrozole combined with gonadotrophins. We have found that highest pregnancy rates are achieved when gonadotrophins are added with Clomephene/Letrozole in IUI cycles.

Out of 30 conceptions, 17 patients were in 21-30 years age group and 13 patients were in 31-40 years age group. Patients who were younger showed higher pregnancy rates then elder patients. 14 conceptions were in couples with 3-5 years of infertility, 11 conceptions were in couples with 6-10 years of infertility, 5 conceptions in more than 10 years of infertility and 1 conception in less than 2 years of infertility. Higher pregnancy rates were found in male factor infertility and unexplained infertility.It seems that natural cycle IUI has no significant beneficial effect over expectant management in case of unexplained infertility." On the other hand the combination of ovarian stimulation and IUI significantly improves live birth rates in couples with unexplained infertility ${ }^{11}$ some prefer double IUI in cases of male factor infertility. However, studies shows no improved pregnancy rates over single IUI. It may increase psychological and financial burden over the couple.

The conception rate of IUI depends on different factors like age of woman, the protocol of ovarian stimulation used, infertility duration, infertility causes, the count and motility of spermatozoa, and other factors. The number of motile sperm available for insemination and especially their 24-hour survival are highly predictive of IUI success. IUI is bypassing the vagina and cervix, so cervical infection or the presence of antibodies or other immunological causes are nullified.

Side effects of mild intensity that occurred in our female patients: 7 patients complained of pain immediately after the intervention, 2 patients required analgesics, 1 patient had nausea, also immediately after the intervention. We had no serious complications. IUI can be accompanied with some complications, from mild to the very serious. Less severe complications include pain (contraction of the uterus), nausea, vomiting and fever. More serious complications include endometritis, adnexa, tubo-ovarian abscess, parametritis and peritonitis.

We had $28.84 \%$ pregnancy rate per patient and $10.79 \%$ pregnancy rates per cycle. Study done by Rojanasakul et al showed $28 \%$ pregnancy rates per patient and $8 \%$ pregnancy rates per cycle. ${ }^{8}$ Similar study performed by A Campana et al," the overall pregnancy rate $18.7 \%$, and the pregnancy rate per cycle $5.6 \%$. The cumulative pregnancy rate calculated by life table analysis showed that $16.0 \%$ of pregnancies occurred in the first three treatment cycles, while the cumulative pregnancy rate was $26.9 \%$ by the sixth cycle." 12

We have satisfactory results for offering IUI as a method of assisted reproduction to infertile couples. Our results are consistence with published data and research

\section{Conclusion}

Infertility should be addressed as problem of society even though it is a problem of couple .This problem is rising as psychological problem also.

In the treatment of infertility, it is been proved that ovarian stimulation in controlled manner with intrauterine insemination is more successful. It is very effective in the known cases of cervical factors, disorders of ovulation, infertility in male, mild endometriosis and most of the unknown infertility causes.

IUI is a low cost, less invasive method. It is easy to learn and can be performed with less equipment compared to other methods of assisted reproduction. So psychological stress of the couple is also less compared to IVF. Numerous reports indicate that the pregnancy rate is much higher if IUI is combined with controlled ovarian stimulation. The success rate of IUI is $10 \%$ $12 \%$ per cycle. Generally 3 - 6 cycles of IUI treatment are attempted before considering other options. Proper patient selection criteria with optimum ovarian stimulation is the key for IUI success.

\section{References}

1. "WHO Infertility". Who.int. 2013-03-19. Retrieved 201306-17.

2. Unisa S. Childlessness in Andhra Pradesh, India: Treatment seeking and Consequences. Reproductive Health Matters. 1999;7:54-65. 
3. Nagaraj A. For a large number of childless couples, life begins in aPetri dish. Indian Express; 2000. Available from:

http://shodhganga.inflibnet.ac.in/bitstream/10603/60567/ 21/21_summary.pdf. [Lastaccessed on 2017 Feb 17].

4. World Health Organization. In Fecundity, Infertility and Childlessness in Developing Countries. DHS

Comparative Reports No. 9. Calverton, Maryland, USA: ORC Macro and the World Health Organization; 2004.

5. Himmel W, Ittner E, Kochen MM, Michelmann HW, Hinney B, Reuter M, Kallerhoff M, Ringert RH (1997). "Voluntary Childlessness and being Childfree". British Journal of General Practice. 47(415):111-8.

PMC 1312893 .PMID 9101672.

6. "ART fact sheet (July 2014)". European Society of Human Reproduction and Embryology. Archived from the original on 4 March 2016.

7. Corsan GH, Kemmann E. The role of superovulation with menotropins in ovulatory infertility: a review. Fertile steril 1991;55:468-77.

8. Jonathan S. Berek. Novak's Gynecology, 2002 by Lippincott Williams \& Wilkins;403.

9. Branigan EF, Estes MA, Muller CH. Advanced semen analysis: a simple screening test to predict intrauterine insemination success. FertilSteril 1999 Mar;71(3):547-51.

10. Rojanasakul A, Suchartwatnachai C, Choktanasiri W, Wongkularb A, Hansinlawat P, Chinsomboon S. Two years' experience of intrauterine insemination for the treatment of infertility. J Med Assoc Thai Aug, 1993;76:8,415-23.

11. Verhulst SM, Cohlen BJ, Hughes E et al. Intra-uterine insemination for unexplained subfertility. Cochrane Database Syst Rev. 2006;(4):CD001838.

12. Campana A, Sakkas D, Stalberg A, Bianchi PG, Comte I, Pache T, Walker D. Intrauterine insemination: evaluation of the results according to the woman's age, sperm quality, total sperm count per insemination and life table analysis. Hum Reprod 1996 Apr;11(4):732-6. 